\title{
Real Time Control of Robotic Arm Using Electromyogram (EMG) Signals
}

\author{
Shital B. Sonone ${ }^{1}$, G. D. Dalvi ${ }^{2}$ \\ ${ }^{1}$ P. R. Pote (Patil) Welfare \& Education Trust's College of Engineering \& Management, Amravati, India \\ ${ }^{2}$ Professor, Electronics \& Telecommunication Engineering, P. R. Pote (Patil) Welfare \& Education Trust's College of Engineering \& \\ Management, Amravati, India
}

\begin{abstract}
Nowadays, the rate of disabled and the people who find difficulties in using their limbs due to age are increasing. A humanassisting robot is a better option for them to overcome this problem. An Electromygraphy (EMG) is a physiological signal that is produced due to the electrical activity when muscle contracts. These signals can be used as control signals for serving the robot. In this project, a robotic arm is controlled using the EMG signals acquired from the forearm of the user. The proposed interface can be used to control a pick and place robotic arm in real time. EMG signals are acquired from the forearm of the user with the help of surface electrodes attached to the user's skin, which avoids bulky interface sensors. Moreover, it is found as the proposed system is robust to muscle fatigue or adjustments of contraction level.
\end{abstract}

Keywords: Electromyographic (EMG) signals, Electrodes, Robotic arm, Mean Absolute Value (MAV) value, variance.

\section{Introduction}

As we know robots find useful in many fields. The humanrobot interface has been proposed in several studies earlier. Most of the previous work proposes complex mechanisms where the user should be trained to map his/her action to the motion desired for the robot. In this project, a new means of control interface is proposed, in which the user performs natural motions with his/her hand. Surface electrodes which are placed on the user's skin record the Electromyography (EMG) activity of the muscles of the forearm. The recorded muscle activity was processed such that they can be used to control the robot arm. Here we use a pick and place edge robotic arm which moves according to the arm movements.

In this project, we propose a methodology for controlling an anthropomorphic robot arm using surface recordings from the muscles of the upper limb, which is robust to time variation of EMG signals. The system architecture was divided into two phases: the training and the real-time operation. During the training phase, the user was instructed to move his/her arm in random patterns with variable speed in the 3-D space. A position tracking system was used to record the arm motion during reaching, while surface EMG electrodes were used to record the activity of nine muscles of the shoulder and the elbow. EMG signals and computed signal features are incorporated into a classification and estimation methodology in order to train a set of models that will be used in real time for arm motion estimation using only EMG recordings. As soon as the training phase had finished, the real-time-operation phase commenced. A control law that utilized the motion estimates was applied to the robot arm actuators. In this phase, the user could teleoperate the robot arm in real time, while he could correct any possible robot deviations since he had visual contact with the robot. The efficacy of the proposed method is assessed through a large number of experiments (four subjects, five sessions per subject, 3.5 min each) during which the users controlled the robot arm in performing random movements in the 3-D space.
EMG signals have often been used as control interfaces for robotic devices specially robotic arms. However, since the musculoskeletal system is very complex and the EMG signals are non-stationary signals, only discrete control has been realized. While going through the earlier studies, some developed an upper-arm EMG-based robot control system using the adaptive neuro-fuzzy inference system (ANFIS) to realize the fuzzy system[2]. Others explained the hand-arm robotic system using haptic technology[7],consists. of data Glove with flex sensors and Micro-Electro Mechanical System (MEMS). Another was a prosthetic finger system based on the EMG signals and uses the Hilbert transform two EMG patterns[3]. In[6], robotic arm with four degrees of freedom is designed in which servomotors are used. Here the input is given using arm made of polycarbonate fitted with potentiometers with a certain angle of rotation. Artemiadis and Kyriakopoulos[8] proposed an EMG-based position and force control scheme for robot arm which had training and real time phases. Continuous models have been built in the past in order to decode arm motion from EMG signals. The Hill-based muscle model[9], whose mathematical formulation can be found in[10] is most used in the literature[11]. However, only a few DOFs were analyzed (i.e., 1 or 2), since the nonlinearity of the model equations and the large number of the unknown parameters for each muscle made the analysis rather difficult. Therefore, random arm motions were never efficiently decoded through EMG signals for the scope of the EMG-based robot control.

Small electrical currents are generated in the muscle fibers before the muscle contraction is produced. These currents are due to the ionic exchange across the membranes of the neural-muscular junctions, which propagate through the resistive surrounding tissues and generate small potential differences. These signals, called electromyographical (EMG), represent fibers contraction, and can be used as a muscular activity indicator and, therefore, be interpreted to control an external electromechanical device. Muscles consist of muscle fibers, activated by motoneurons. Impulses 


\section{International Journal of Science and Research (IJSR) \\ ISSN (Online): 2319-7064}

Index Copernicus Value (2013): 6.14 | Impact Factor (2014): 5.611

from the spinal cord arrive to the motoneuron and trigger a group of several muscle fibers, called motor unit. To produce a movement, each muscle fiber composing the muscle contracts, carries the contraction to the whole muscle and achieves the desired action. In most of the cases, even for a fine movement, several muscles are simultaneously involved to accomplish that action.

In this project, real time control of robotic arm using surface EMG signals are proposed. For simplicity only two movements are taken into consideration; flexion and extension. The signals are taken from the forearm of the user and processed to serve as control signals for the robotic arm. At first a training process was done by collecting EMG signals from the forearm of different persons who are having similar features (height, weight etc). After processing these signals calculated the range. In the next step robotic arm was controlled by the EMG signals in real time.

\section{System Design}

\section{A. Problem definition}

There is no doubt that the EMG signals are non stationary signals which are complex. However, they can be used for interfacing the human with the robotic devices. Here, a human-robotic interface is proposed using the EMG signals. The EMG signals from the forearm is used for controlling the pick and place robotic arm. For simplifying the work only four movements are considered, that is, flexion, extension, hand opening and hand closing. An EMG signal represents the electrical activity of the muscle due to the potential difference when the electrode is placed as the muscle is contracted. The amplitude of the electrical signal generated by the muscle activation will depend upon the force applied to move the joint, the velocity of muscle contraction and the angle of muscle pull when it contracts. Fig. 1 represents the proposed model for the robotic arm control. Here, a robotic arm is controlled using the EMG signals in real time. For accomplishing this task,as in Fig.1, the initial step is to acquire the EMG signals from the fore arm of a person. For this purpose, surface electrodes are used. EMG signals are collected from different persons. They are asked to do the two motions, flexion and extension, each for a time duration of 10 seconds and about 10 samples are taken. These signals are then given to the microcontroller. The corresponding digital values are given to the robotic arm and thus it can be controlled in real time.
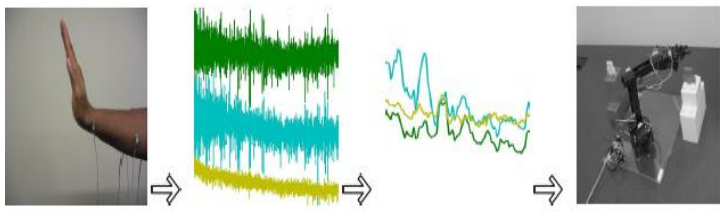

Figure 1: Proposed System Model

\section{B. Gestures for robot arm control}

Fig. 2 shows a list of the actions we use to control each degree of freedom in the robotic arm. Our goal is to map gross actions (distinguishable via forearm musculature) at the wrist to commands for the robotic arm. EMG signals are the expression of impulses which are initially generated in the central nervous system and then travel to their final destination, where they produce the desired result. The potential differences generated in muscles due to these impulses not only generate muscular contraction, but also propagate to the surrounding tissues, which make it possible to measure them by applying specially designed electrodes. There are two main kinds of electrodes that might be used for measuring EMG signals: surface and inserted. Inserted electrodes are very thin wires or needles that are inserted inside muscles and, due to their proximity to the signal origin, allow acquiring precise and high quality signals. The problems about them are many; they could be painful, are not removable without surgery, and there are no records about their time duration. Due to those factors we chose surface electrodes, in particular bipolar electrodes, to measure the voltage difference between two specific points with two electrodes connected to the inputs of a differential amplifier. Since the muscles responsible of hand motionare deeply placed on the forearm, when measuring the myoelectric signals with surface electrodes it is not possible to exclusively register the signal of the involved muscle, but we obtain the superimposition of every signal generated between the point of interest and the detection surface. This phenomenon is known as "Cross-talk" [15]. By using bipolar electrodes, the "common" information registered by both electrodes is mostly eliminated by a differential amplifier. Anyway, due to the geometrical configuration between both electrodes, it is not necessarily true that the signal registered in each one of them encloses Cross-Talk due to the same signals as the other one; that is why the output of the differential amplifier doesn't necessarily represents an exact subtraction of both signals.

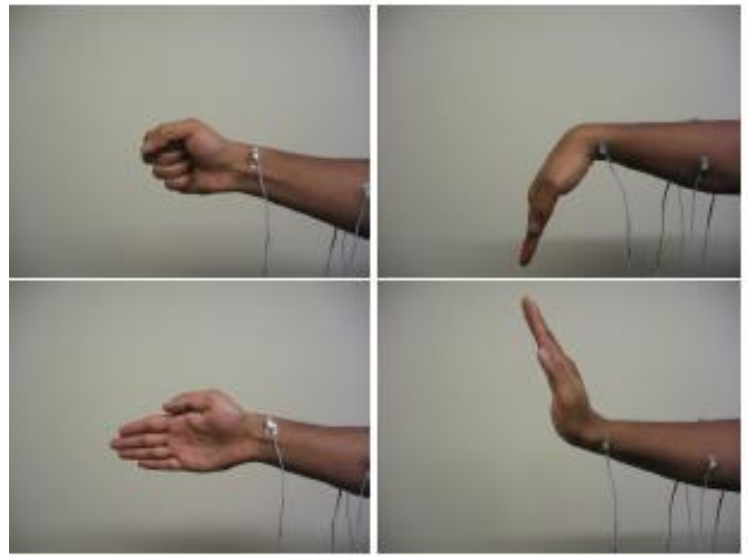

Figure 2: Static hand gestures choosen for controlling the robotic arm

\section{Electrode placement}

Our choice of electrode positions was designed to make theinterpretation of the signal as intuitive as possible and as reproducible from subject to subject as possible. While no electrode position will isolate a single muscle, placing a given electrode on the skin above a given superficial muscle should ensure that the largest contribution to the signal from that location is from the desired muscle. The muscles of the deep layer will contribute to the signal, as will other surrounding muscles. Since our goal is classification into a discrete set of actions, and not the study of individual muscles, we rely on the classifier to extract the important 


\section{International Journal of Science and Research (IJSR) \\ ISSN (Online): 2319-7064}

Index Copernicus Value (2013): 6.14 | Impact Factor (2014): 5.611

components for each class from this mixture of information in each electrode channel.

The muscles we chose, and their relevant functions are listed below: (1) Brachioradialis- flexion of the forearm, (2) Extensor carpi ulnaris- extension and adduction of hand at the wrist, (3) Pronator Teres- pronation and elbow flexion (which was not a movement we incorporated), (4) Extensor Communis Digitorum- extension of fingers at the metacarpophalangeal joints and extension of wrist at forearm, (5) Flexor Carpi Radialis- flexion and abduction of hand at wrist, (6) Anconeus- antagonistic activity during pronation of the forearm, (7) Pronator Quadratus- initiates pronation. The combination of these muscles in coordination span the movements that we classify and map to arm control. Because of redundancy amongst the actions of these muscles as well as the redundancy amongst deeper muscles whose signals are measured in conjunction, our channels may contain correlations. This redundancy of information leads to a more robust classifier that can handle the unavoidable variations in electrode placement and quality of EMG recordings across subjects and sessions.

We use single electrodes, in contrast to the differential pair at each recording site traditionally used in the literature (Deluca 1997). Instead we use an eighth electrode on the upper arm as a reference for the other electrodes.

\section{Feature Extraction}

Wavelets are one of the several mathematical transformations to extract information from signals. The particularity of Wavelet Transformation is that its result is a signal representation in the time frequency space, i.e., it is possible to know when a certain phenomenon occurs with a specific frequency [12].

Wavelet is a series decomposition of the signal in a set of functions _ $(\mathrm{t})$, that are different both in the scale factor (a) and in the time shift $(b)$.

$$
W f(a, b)=\int_{-\infty}^{+\infty} f(t) \frac{1}{\sqrt{2}} \oint\left(\frac{t-b}{a}\right) d t
$$

We chose a temporal approach, where features are directly extracted from the temporal sequence. According to [8] we have to characterize the time sequence with some parameters; we extract the following four statistical features:

1)Mean Absolute Value(MAV): The MAV value can be defined as the statistical measure of the magnitude of a varying quantity, here, the EMG signals. It is the arithmetic mean of the squares of the set of values. This will help to find the digital values required for the robotic arm control. The MAV value is calculated for the samples taken from different persons. Then the range of the value is computed. Let $\mathrm{xk}$ represent the samples then MAV is given by,

$$
\mathrm{Xi}=\frac{1}{\mathrm{~N}} \sum_{\mathrm{k}=1}^{\mathrm{N}}|\mathrm{xk}|
$$

2)Difference between the MAV of two samples: $\Delta \mathrm{Xi}=\mathrm{Xi}+1-\mathrm{Xi}$
3) Length of the Signal

$$
\mathrm{lo}_{\mathrm{o}}=\sum_{\mathrm{k}=1}^{\mathrm{N}}|\mathrm{Xk}-\mathrm{Xk}-1|
$$

\section{4) Variance:}

Variance specifies the inequalities between the samples. It represents the contraction levels in the signals. It is usually represented as,

$$
\frac{1}{N-1} \sqrt{\frac{1}{N}} \sum_{n=0}^{N-1} X n / 2
$$

\section{E. Overview}

For accomplishing the task of human-robot interface, the initial step is to acquire the EMG signals from the fore arm of a person. For this purpose, surface electrodes are used. These surface electrodes are attached to the fore arm and thereby extracting the signals as shown in fig.2. It filters and amplifies the EMG signals. We can acquire these signals using the microcontroller. After acquiring EMG signals it should be processed. After converting it to digital values it should be given to the robotic arm. Here, microcontroller is used to give the digital values corresponding to the EMG signals to the driver circuit. The driver circuit is made of L293D. The robotic arm consists of five motors. Each motor can be driven using the driver circuit by giving the digital values. Thus according to the movement of human arm the robotic arm is controlled virtually in real time.

\section{Conclusion}

Here, we were able to control the robotic arm using the EMG signals acquired from the fore arm of the person. By extracting the required features from the signals the arm is controlled. The EMG signals were acquired from the fore arm for the movements flexion and extension of different persons. This was done by attaching surface electrodes to the fore arm with the help of the microcontroller. The samples were taken and features like RMS and Variance were extracted. These features were used to produce the control signals. The OWI-535 EDGE robotic arm was controlled by giving digital values from the microcontroller through the L293D driver circuit. In this way the microcontroller gives the control signals to the driver circuit and thereby the robotic arm can be controlled.

\section{Acknowledgment}

We would like to acknowledge the Faculties of Electronics \& Telecommunications Department, P. R. Pote(Patil) Welfare \& Education Trust's College of Engineering \& Management, Amravati for their support. I Miss. Shital B. Sonone specially want to thank my guide Prof. G.D. Dalvi for their guidance in field of Bio-medical Engineering and constant encouragement towards the project work. 


\section{International Journal of Science and Research (IJSR) \\ ISSN (Online): 2319-7064}

Index Copernicus Value (2013): 6.14 | Impact Factor (2014): 5.611

\section{References}

[1] Panagiotis K. Artemiadis and Kostas Kyriakopoulos, EMG-Based Control of a Robot Arm Using Low Dimensional Embeddings, IEEE Transactions on robotics, Vol. 26, Issue No. 2, April 2010.

[2] Hsiu-Jen Liu and Kuu-Young Young, An adaptive upper-arm EMG-based Robot control system International Journal of Fuzzy Systems, Vol. 12, Issue No. 3,Pg No.181-189 September 2010.

[3] Amanpreet Kaur and Gagandeep Kaur, The Implementation OF Prosthetic Index Finger Based On EMG Signals, International Journal Of Computational Engineering Research,Vol. 2,Issue No.3,Pg.No.898900, May-June 2012.

[4] Kyriakopoulos and Panagiotis K. Artemiadis," An EMG-Based Robot Control Scheme Robotust to TimeVarying EMG Signal Features", IEEE Transactions on information technology in biomedicine Vol.14,Issue No.3,Pg No.582-588,May 2010.

[5] O. Fukuda, T. Tsuji, M. Kaneko, and A. Otsuka, "A human-assisting manipulator teleoperated by EMG signals and arm motions," IEEE Transactions Robot. Autom., vol. 19 no.2, April 2010.

[6] A.Rama Krishna,G. Sowmya Bala , A.S.C.S. Sastry ,B. Bhanu Prakash Sarma and Gokul Sai Alla,," Design And Implementation Of A Robotic Arm Based On Haptic Technology", International Journal of Engineering Research and Applications (IJERA) Vol.No.2, Issue No.3,Pg No..3098-3103,May-June 2012.

[7] Niji Johnson and P.Sivasankar Rajamani, "A hand-arm robotic system for autonomous motion control, International Journal of Emerging trends in Engineering and Development Vol.No3 Issue No. 2,Pg No.52-59,April-2012.

[8] Anthony Mandow, Alfonso J. García-Cerezo, "Using LEGO NXT Mobile Robots With LabVIEW for Undergraduate Courses on Mechatronics", IEEE Transactions on education, $\mathrm{Vol}$ No.54,Issue No.1,Pg No.41-47,February 2011

[9] A. V. Hill, "The heat of shortening and the dynamic constants of muscle," Proc. R. Soc. Lond. Biol., vol. 126, pp. 136-195, 1938.

[10] F. E. Zajac, "Muscle and tendon: Properties, models, scaling, and application to biomechanics and motor control," CRC Crit. Rev. Biomed. Eng., vol. 17, pp. 359-411, 1986.

[11] E.Cavallaro, J. Rosen, J. C. Perry, S.Burns, and B. Hannaford, "Hill-based model as a myoprocessor for a neural controlled powered exoskeleton arm parameters optimization," in Proc. IEEE Int. Conf. Robot. Autom., 2005, pp. 4514-4519.

[12] S.G. Mallat, A theory for multiresolution signal decomposition Thewavelet representation, IEEE TransPAMI 11 (1989), 974-693.

[13] H. H. Szu, B. Telfer and S. Kadambe, Neural network adaptive wavelet for signal representation and classification, Optical Engineering 31 (1992), 19071916.
[14] B. Hudgins, P. Parker and R. N. Scott, A new strategy for multifunction myoelectric control, IEEE Trans BME 40 (1993), 82-94.

[15] J.W. Morrenhof and H.J. Abbink, Cross-correlation and cross-talk in surface electromyography, Electromyogr Clin Neurophysiol 25 (1985), 73-79.

[16] O. Fukuda, T. Tsuji, M. Kaneko, and A. Otsuka, “A human-assisting manipulator teleoperated by EMG signals and arm motions," IEEE Trans. Robot. Autom., vol. 19, no. 2, pp. 210-222, Apr. 2003.

[17] Giuseppina Gini, Matteo Arvetti, Ian Somlai and Michele Folgheraiter,"Acquisition and analysys of EMG signals trecognize multiple hand movements for prosthetic application",Applied Biomics and Biomechanics 9(2012) 145-155 DOI 10.3233/ABB2011-0024.

\section{Author Profile}

Shital Bhimrao Sonone received Bachlor of Technology in Electronics and Telecommunication from Government College of Engineering, Amravati \& persuing Master of Engineering in Electronics \& Telecommunication Engineering from P. R. Pote (Patil) Welfare \& Education Trust's College of Engineering \& Management, Amravati, India.

Prof. Gopal D. Dalvi received M. Tech degree from SSCOE\&T College, Durg. He is now a Principal of P. R. Pote (Patil) Welfare \& Education Trust's College of Polytechnic \& Management, Amravati, India. 